\title{
A Complexity Reduced Non-Uniform Generalized Memory Polynomial Model for Nonlinear Power Amplifier Behavioural Modeling
}

\author{
Anqi Hu, Declan Gerard Byrne, Ronan Farrell, John Dooley \\ Department of Electronic Engineering. National University of Ireland, Maynooth (NUIM)
}

\begin{abstract}
Power amplifiers are widely employed electronic devices in various fields such as mobile networks and radio frequency (RF) transceivers. To achieve efficient operations, power amplifiers can often suffer from nonlinearity problems. This problem can be mitigated through the use of linearization techniques, such as digital predistortion, regarded as the most promising solution to power amplifier linearization. Behavioural modeling is a substantial part of the digital predistortion, responsible for acquiring the coefficients that are necessary to linearize the power amplifier. A Complex Reduced Non-Uniform Generalized Memory Polynomial model was proposed to reach comparable performance of accuracy as Memory Polynomial Model with reduced complexities. The proposed model was tested with a $5 \mathrm{MHz}$ LTE signal measured at the input and output of a Doherty PA under different conditions of nonlinearities, memory effects and attenuations as well as PA working powers. It can be observed that the proposed model shows superior accuracy at low complexities, when the PA has higher levels of nonlinearity and memory depth while still maintaining low complexities. Over $60 \%$ of coefficients reduction could be reached at the same level of accuracy compared to the MP model.
\end{abstract}

Keywords-- Accuracy, Complexity, GMP model, MP model

\section{INTRODUCTION}

A major source of the nonlinear distortion for wireless communication systems arises from Power Amplifiers (PA) [1]. As a widely applied device in wireless communication links, such as in Radio Frequency (RF) transceivers [2] for wireless communication systems, mobile cellular networks [3] and digital video broadcasting applications [4]. Particularly in the wireless communication systems [3], [5], one of the main objectives in wireless communication system is to enhance the spectral efficiency within the allocated transmitting bandwidth [1]. However, the widely employed signals such as Wideband Code Division Multiple Access (WCDMA) in 3G mobile networks and Orthogonal Frequency Division Multiple Access (OFDMA) in 4G mobile systems are vulnerable to the nonlinear distortions due to RF PAs [3].

Digital Predistortion (DPD) has been widely accepted as a solution to the nonlinearity problems. The main objective for these approaches is to maintain highly linear performance, while optimizing the power efficiency performance of the PA [6]. The architecture of a linearized system is composed of two cascaded nonlinear subsystems. The first subsystem is a
DPD block which implements a nonlinear function with inverse envelope response of the PA's nonlinear behavior. The second subsystem is the PA [1].

Behavioural Modeling of PAs is an essential step of DPD [7]. The predistortion coefficients identification is performed in conjunction with a behavioural model acquired using a PA's measured data. Nonlinear behavioural modelling has been accomplished using models based on the Volterra series. Volterra models are widely known for their superiority accuracy while characterizing PA features[8]. However, with the increase of nonlinear order and memory depth, model complexity increases rapidly[1]. Hence, Volterra model is also not ideal for characterizing PAs with light nonlinearity and/or weak memory effect, due to a likelihood overfitting. Several complexity reduced (CR) Volterra models have been proposed in previous literature including the Memory Polynomial (MP) and the Generalized Memory Polynomial (GMP) [6], [8], [9], [10], [11]. These models are relatively compact but often employ coefficients of insignificant contribution.

In this case, multiple complex reduced (CR) Volterra models were modified and proposed for the application in wideband systems including (MP) model [6], [9], Generalized Memory Polynomial (GMP) model and various modified or simplified version [1] as well as Dynamic deviation reduction based Volterra (DDR-Volterra) model [12].

A simplified GMP model for wideband RF PAs was proposed in paper [1] to achieve lower complexity while at the price of reduced accuracy which was compensated later by the sub-block of nonlinear memory effect (NME). Hence, compared to GMP model, the performance was comparable with fewer coefficients and reduced complexity. Another paper [8] proposed a modified version of Volterra series model with high accuracy of predicted output signals both in time domain and frequency domain. In that work, a quasimemoryless model is extracted to represent nonlinearities, while the memory effects were characterized by parallelly adding the estimated coefficients for Volterra filters.

The GMP model is regarded as one of the most accurate models for the number of coefficients. The computational complexity for GMP is higher than that of the MP model but lower than the Volterra model [13]. In this paper, a modified 
version of CR non-uniform GMP model is proposed, which can maintain the same level of performance as the MP model while employing less coefficients. To deal with the nonlinear systems with severe nonlinearities and memory effects, the superiority of the proposed model can be clearly observed.

\section{THE MODEL DESCRIPTION AND COMPARISON}

\section{A. Memory Polynomial Model}

A memory polynomial (MP) model can be obtained by selecting a subset of coefficients from the Volterra series model, in particular, its diagonal terms. In other words, if all the cross-terms are removed from the Volterra series, the resulting model is an MP model is extracted and defined as in (1) [13].

$$
y_{M P}=\sum_{m=0}^{M} \sum_{k=1}^{N} w_{m k} \cdot x(n-m) \cdot|x(n-m)|^{k-1}
$$

Where $x$ and $y_{M P}$ represent the complex input signal and complex output signal of MP model respectively. The symbol $w$ denotes the model's coefficients. $M$ and $N$ denote the memory depth and nonlinearity order respectively.

\section{B. The proposed Complexity Reduced GMP Model}

The GMP model could be obtained by augmenting the memory polynomial model with additional cross-terms resulting from the leading and lagging terms of complex input signals. Equation (2) can be used to describe the formation of the GMP model [13].

$$
\begin{aligned}
& y_{G M P}=\sum_{m=0}^{M_{a}} \sum_{k=1}^{N_{a}} a_{m k} \cdot x(n-m) \cdot|x(n-m)|^{k-1} \\
& +\sum_{m=0}^{M_{b}} \sum_{k=2}^{N_{b}} \sum_{p=1}^{P} b_{m k p} \cdot x(n-m) \cdot|x(n-m-p)|^{k-1} \\
& +\sum_{m=0}^{M_{c}} \sum_{k=2}^{N_{c}} \sum_{q=1}^{Q} c_{m k q} \cdot x(n-m) \cdot|x(n-m+q)|^{k-1}
\end{aligned}
$$

Where the first polynomial function refers to the timealigned input signal samples whose memory depth and nonlinearity order are $M_{a}$ and $N_{a}$ respectively; the second polynomial function introduces cross-terms between the input signal and its lagging values of envelope with memory depth of $M_{b}$ and nonlinearity order of $N_{b}$ and lagging terms up to $P^{\text {th }}$ order; similarly, the leading values of input signal's envelope are combined with time-aligned signal up to $Q^{\text {th }}$ order with memory depth of $M_{c}$ and nonlinearity order of $N_{c}$. In addition, $a_{m k}, b_{m k p}$ and $c_{m k q}$ represent the model coefficients of time-aligned terms, lagging cross-terms and leading cross-terms.

All the instantaneous time-aligned complex input signal, leading terms and lagging terms have equal nonlinear orders, which provides a uniform model structure to GMP model. Nevertheless, in the proposed Non-Uniform GMP model, the nonlinearity orders of the branches are unequal. Certain terms of the time-aligned terms and cross-terms were selected and abandoned if they provide an insufficient contribution to the behavioral model. Hence, compared to the conventional GMP model, a significant decrease in the number of coefficients can be accomplished for the non-uniform general memory polynomial model. The complexity reduction is desired in the broadband systems where the memory effect is more severe.

\section{MODEL IDENTIFICATION AND COMPARISON}

\section{A. Identification of NUGMP and MP Model}

As described above, the proposed NUGMP model is a modified non-uniform Generalized Memory Polynomial model with reduced complexity. It contains cross-terms at different memory depths and nonlinearity orders while certain terms were abandoned due to negligible contribution to the behavioral model output. To identify the PA feature using NUGMP model and MP model, initially, a model structure matrix was constructed in MATLAB with all combinations of terms for a given memory depth $m$ and nonlinearity order $n$. Then, both the models were trained using a least squares algorithm. Finally, the coefficients were extracted based on the training data which are parts of the raw data set of inputs and outputs measured from the experimentally measured PA signals. Once the PA models using the modified NUGMP and MP model were successfully constructed, the next procedure is to obtain the optimal values of $m$ and $n$ as well as the resulting coefficients by iteration. Finally, an independent portion of raw data was used as an independent testing signal to validate the accuracy and complexity improvements of NUGMP model upon MP model.

\section{B. Comparison of Complexity}

It is commonly known that the structure of GMP model contains more terms than the MP model as a result of the introduction of cross-terms. However, the modified NonUniform GMP contains less terms than the GMP model while maintain sufficient accuracy of the behavioural modeling performance. Compared to the widely applied MP model, the proposed NUGMP model could realize comparable accuracy of performance while using reduced number of coefficients. This feature was managed by intelligently selecting the terms from complete GMP model and abandoning the terms whose contributions to the behavioral model were neglectable.

\section{EXPERIMENTAL RESULTS}

\section{A. Measurement set up}

In order to validate the proposed NUGMP model and compare its performance to the MP model, three datasets of input and output signals obtained from a GaN-SiC Doherty amplifier design working at different levels of attenuation and output power were applied to test the model accuracy. The first dataset was collected at PA power of $30.81 \mathrm{dBm}$ with $-7 \mathrm{~dB}$ of attenuation; the second dataset and the third data set correspond to the working power of $28.02 \mathrm{dBm}$ with $-10 \mathrm{~dB}$ 
of attenuation and $24.68 \mathrm{dBm}$ with $-14 \mathrm{~dB}$ of attenuation respectively. For the purpose of observing the superiority of the modified NUGMP model compared to the conventional MP model, three datasets experience different levels of nonlinearity, memory effects and attenuation.

A 5MHz LTE standard signals was selected as the testing signal in this experiment due to limitations in the output signal measurement bandwidth. Measurements of inputs and outputs were recorded and induced to MATLAB directly for the use of constructing PA models. In the end, the estimated outputs from the constructed PA model of NUGMP and MP were compared together with the original dataset.

\section{B. Behavioural Modeling Results and Evaluation}

With reference to the original data from test bench, the performance results of the modified NUGMP model were described from three aspects of time domain, frequency domain and PA feature characterizations, MP model results were also contained for comparison.

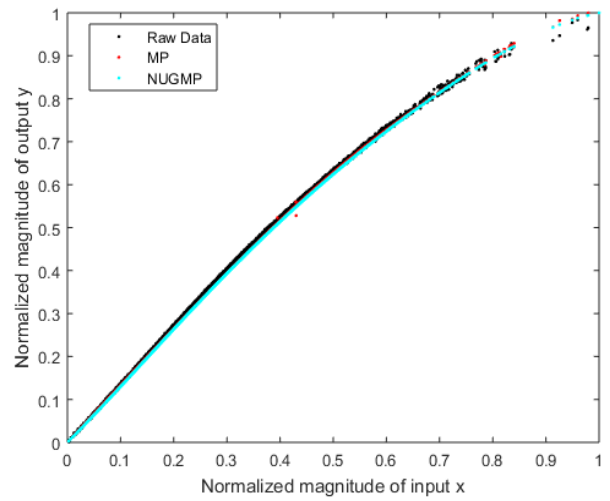

Fig. 1. AM/AM PA feature extracted from raw data set of actual PA, Memory Polynomial Model and Non-Uniform General Memory Polynomial Model $\left(\mathrm{P}_{\text {out }}=30.81 \mathrm{dBm},-7 \mathrm{~dB}\right.$ attenuation $)$

As shown in Figure 1, the black, red and cyan curve stands for the AM/AM feature of PA extracted from raw data set, MP model and NUGMP model respectively. The raw data were obtained at $30.81 \mathrm{dBm}$ working power with $-7 \mathrm{~dB}$ attenuation which shows severe nonlinearities and memory effects. Notice that the magnitude of inputs and outputs data were normalized for clearer comparison and observation. Both the fitting results of MP model and NUGMP model reaches quite close to the reference of raw data. However, the memory effects of constructed model were to some degree alleviated. Specific error difference would be calculated and discussed below.

The selection of the optimal values of memory depth $m$ and nonlinearity order $n$ were acquired by iteration from 1 to 10 . For the case of $30.81 \mathrm{dBm}$ output PA power with $-7 \mathrm{~dB}$ attenuation, the optimal values of $m$ and $n$ for MP model and NUGMP model were selected as $m=5, n=3$ and $m=1, n=5$ respectively. Under these circumstances, the corresponding number of coefficients developed by MP model and NUGMP model were 20 and 7 respectively. As a result, the model complexity of the proposed NUGMP model was greatly reduced compared to the frequently used MP model. In the optimal case, the fitting performances were evaluated through the Mean Squared Error (MSE), which can be calculated by equation (3)[13].

$$
M S E=\frac{1}{N} \sum_{i}(y-\hat{y}) *(y-\hat{y})^{\prime}
$$

Where $N$ represents for the number of data points stored in the signal. The symbol $y$ and $\hat{y}$ represents for the actual PA output signal and the estimated output of constructed models. Notice that the test signal is complex signal, hence the product of error signal and its conjugate is the squared magnitude of the error.

$$
\begin{array}{rrrrrr}
10.0000 & 5.0000 & -40.3966 & 10.0000 & 5.0000 & -20.9622 \\
10.0000 & 6.0000 & -40.2986 & 10.0000 & 6.0000 & -20.8281 \\
10.0000 & 7.0000 & -40.4276 & 10.0000 & 7.0000 & -21.1442 \\
10.0000 & 8.0000 & -40.3403 & 10.0000 & 8.0000 & -21.1997 \\
10.0000 & 9.0000 & -40.4123 & 10.0000 & 9.0000 & -21.2053 \\
10.0000 & 10.0000 & -40.0126 & 10.0000 & 10.0000 & -21.2053 \\
\text { best: } 5 & 3 & -43.0294 & \text { best: } 1 & & \\
& \text { (a) } & & & \text { (b) } & -41.9612
\end{array}
$$

Fig. 2. Optimal case of (a) $\mathrm{m}=5, \mathrm{n}=3$ with $\mathrm{MSE}=-43.0294 \mathrm{~dB}$ for MP model $(\mathrm{b}) \mathrm{m}=1, \mathrm{n}=5$ with MSE $=-41.9612 \mathrm{~dB}$ for NUGMP model at $\mathrm{P}_{\text {out }}=30.81 \mathrm{dBm}$ and $-7 \mathrm{~dB}$ attenuation.

Figure 2 (a) and (b) give the optimal values of memory depth $m$ and nonlinearity order $n$ extracted based on the minimized MSE value. Given that the values of the MSE for MP model and NUGMP model are quite close, which indicates similar levels of performance of the two models is reached. The complexity of NUGMP model with 7 coefficients is much less than the MP model whose number of coefficients is 20 .

From the perspective of frequency domain and time domain the relative accuracy of power amplifier output signal characterization can be observed in Figure 3 and Figure 4.

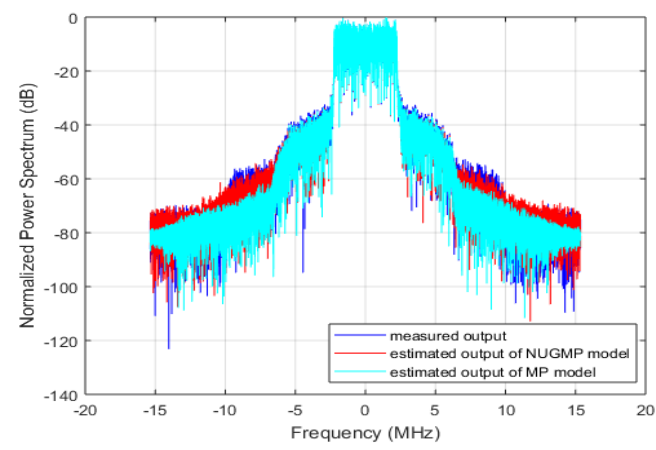

Fig. 3. Frequency Spectrum of measured PA output, estimated output of MP model and NUGMP model ( $\mathrm{P}_{\text {out }}=30.81 \mathrm{dBm},-7 \mathrm{~dB}$ attenuation)

As presented in Figure 3, three curves in blue, red and cyan represent for the frequency spectrum of actual PA output signal with the bandwidth of $5 \mathrm{MHz}$ measured at the test bench, estimated output of the proposed NUGMP model and conventional MP model respectively at their optimal cases. It is noticeable that the estimated output of the MP model plotted in cyan curve is worse than the estimated output from 
NUGMP model plotted in red curve. Although cyan curve has close match with the actual output signal at 3rd order within the range of $-7.5 \mathrm{MHz}$ to $7.5 \mathrm{MHz}$. Nevertheless, the frequency components at higher order were mismatched. It is reasonable to deduce that the mismatch was due to the choice of nonlinearity order of 3 as the optimal values. Therefore,

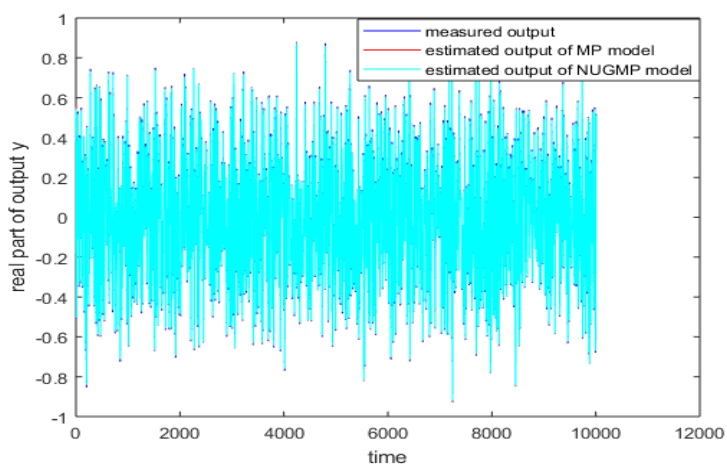

(a)

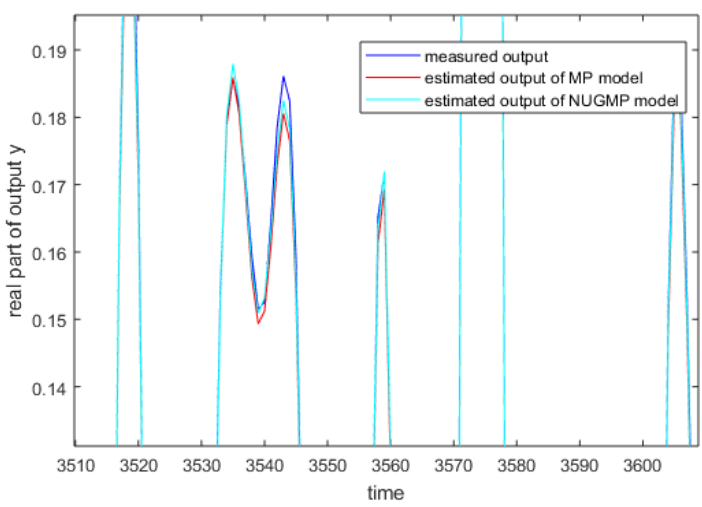

(b)

Fig.4. Comparison of 2 fitting curves from MP model and NUGMP model with actual PA Output signal curve. (a) Time domain Signals; (b) Zoom in version of (a).

frequency components at higher order could not be recovered completely. The selection of the optimal case was based on the MSE value calculated between the magnitude of raw output and estimated output. One of the possible explanations is that low error could be reached with close matching in time domain but might lead to unexpected losses of frequency components at higher orders. Such kind of problems were better avoided in the new NUGMP model fitting process.

As illustrated in Figure 4, both the estimated output signals of NUGMP model plotted in cyan curve and MP model plotted in red curve shows sufficient fitness to the measured raw data. However, mismatches were unavoidable at its peaks. The closeness of two models at signal peaks were nondeterministic and these two models reach their optimal matching in a competitive manner.

As for the testing results of two other data sets worked at $28.02 \mathrm{dBm}$ output power with $-10 \mathrm{~dB}$ of attenuations and $24.68 \mathrm{dBm}$ output power with $-14 \mathrm{~dB}$ of attenuations shows similar features and problems as the case of $30.81 \mathrm{dBm}$ output

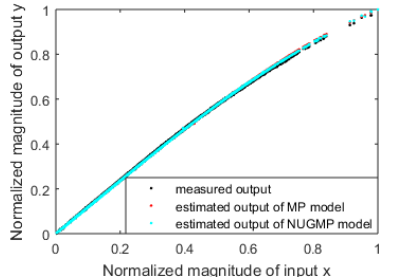

(a)

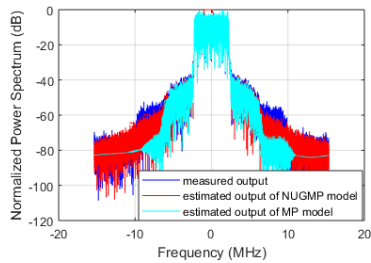

(c)

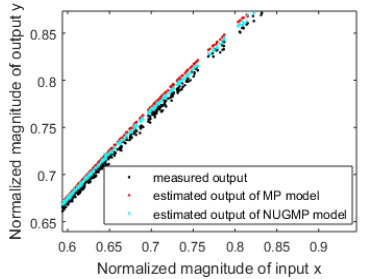

(b)

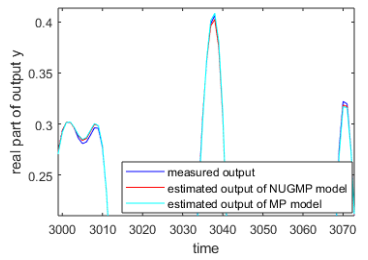

(d)
Fig.5. Comparison of the performance Results among NUGMP model, MP model and actual PA (at $\mathrm{P}_{\text {out }}=28.02 \mathrm{dBm}$ with -10 attenuation). (a) AM/AM characterization of PA; (b) Zoom-in version of figure(a); (c)Frequency spectrum of output signals; (d) Output signals in time domain.

power with $-7 \mathrm{~dB}$ of attenuation. Detailed information could refer to the Figure 5 and Figure 6 including the comparisons of raw PA outputs, estimated outputs of the proposed NUGMP model and conventional MP model in the aspect of time domain and frequency domain as well as AM/AM characterizations with MSE calculations.

As shown in Figure 5, the performance results of the resulting PA model were plotted under the optimal case corresponding to their model. For the proposed NUGMP model, $m=3, n=1$ were selected with $\mathrm{MSE}=-47.7087 \mathrm{~dB}$; for the conventional MP model, $m=3, n=5$ were selected with MSE $=-47.0619 \mathrm{~dB}$. As discussed and concluded in the previous case, the MP model and NUGMP model share similar level of behavioral modeling performance while the complexity of NUGMP

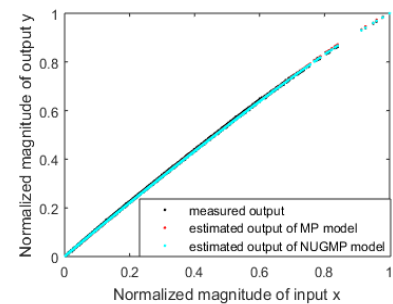

(a)

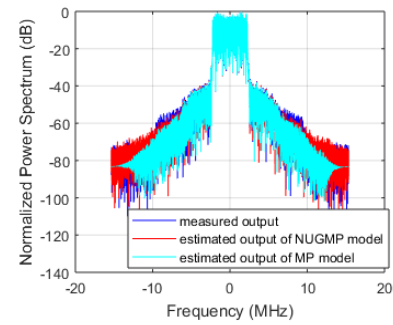

(c)

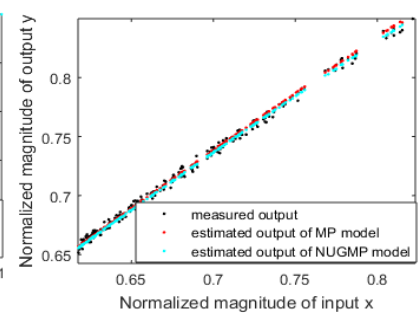

(b)

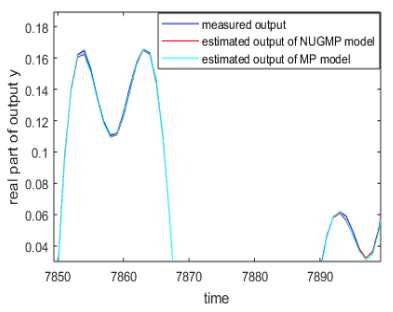

(d)
Fig. 6. Comparison of the performance Results among NUGMP model, MP model and actual PA (at $\mathrm{P}_{\text {out }}=24.68 \mathrm{dBm}$ with -14 attenuation). (a) AM/AM characterization of PA; (b) Zoom-in version of figure(a); (c)Frequency spectrum of output signals; (d) Output signals in time domain. 
model (16 coefficients) was less than the MP model (18 coefficients). One of the possible explanations is that due to the extra cross-terms utilized in the modified NUGMP model, it can reach same level of performance compared to MP model with less demands of memory depths and nonlinearity orders, which reduces the complexity greatly while maintaining sufficient performance.

Other testing results were displayed in Figure 6 for further reference. The PA working power was set to be $24.68 \mathrm{dBm}$ with $-14 \mathrm{~dB}$ of attenuations. Optimal cases for MP model and NUGMP model were selected as $m=4, n=1$ with MSE = $49.2391 \mathrm{~dB}$ and $m=1, n=3$ with $\mathrm{MSE}=-48.3034 \mathrm{~dB}$ respectively. Coefficient taps of MP and NUGMP models are 8 and 5 respectively. The performance results and complexity differences cope with the conclusion discussed in previous conditions.

\section{CONCLUSION}

In summary, a modified Complexity Reduced General Memory Polynomial model was proposed and validated with the comparison of the frequently used Memorial Polynomial. The constructed models were validated with a $5 \mathrm{MHz}$ LTE test signal based on the experimentally measured datasets of input and output from a Doherty PA. Three datasets from different levels of nonlinearities, memory depth and attenuations were tested to validate the accuracy and complexity of the proposed model compared with MP model. For the modified CR non-uniform GMP model, it can be concluded that comparable performance of behavioral modeling for PA was achieved with less model coefficients which implies lower complexity than MP model. Furthermore, with the growing nonlinearities and memory depth, the MP model might suffer fidelity loss at higher frequency components while the modified NUGMP model adapts better to the situation with improved performance resultant from the introduction of the cross-terms under different memory depth.

\section{ACKNOWLEDGMENT}

This publication has emanated from research conducted with the financial support of Science Foundation Ireland (SFI) and is co-funded under the European Regional Development Fund under Grant Number 13/RC/2077.

\section{REFERENCES}

[1] Y. J. Liu, J. Zhou, W. Chen, and B. H. Zhou, "A robust augmented complexity-reduced generalized memory polynomial for wideband RF power amplifiers," IEEE Trans. Ind. Electron., vol. 61, no. 5, pp. 2389-2401, 2014.

[2] Y. Jin and F. F. Dai, "Impact of transceiver RFIC impairments on MIMO system performance," IEEE Trans. Ind. Electron., vol. 59, no. 1, pp. 538-549, 2012.

[3] Q. Zhang and W. Chen, "Digital predistortion for 5G wideband power amplifiers using multiple band-limited feedback signals," 89th ARFTG Microw. Meas. Conf. Adv. Technol. Commun. ARFTG 2017, pp. 1-4, 2017.

[4] E. Soto, R. Perez, J. J. Rodriguez-Andina, J. L. Mato, M. Pereira, and J. Farina, "Distortion Mitigation in RF Power Amplifiers Through FPGA-Based Amplitude and Phase Predistortion," IEEE
Trans. Ind. Electron., vol. 55, no. 11, pp. 4085-4093, 2008.

[5] W. Chen, J. Zhou, B.-H. Zhou, F. M. Ghannouchi, and Y.-J. Liu, "Digital Predistortion for Concurrent Dual-Band Transmitters Using 2-D Modified Memory Polynomials," IEEE Trans. Microw. Theory Tech., vol. 61, no. 1, pp. 281-290, 2012.

[6] Q. Zhang, Y. Liu, J. Zhou, and W. Chen, "A complexity-reduced band-limited memory polynomial behavioral model for wideband power amplifier," 2015 IEEE Int. Wirel. Symp. IWS 2015, 2015.

[7] S. Zhang, G. Su, X. Chen, and W. Chen, "Extraction of wideband behavioral model of power amplifier with multi groups of narrow band signals," 2014 IEEE Int. Wirel. Symp. IWS 2014, pp. 0-3, 2014.

[8] J. Dooley, B. O'Brien, and T. J. Brazil, "Behavioural modelling of RF power amplifiers using modified Volterra series in the time domain," pp. 169-174, 2004.

[9] Q. Zhang, Y. Liu, J. Zhou, S. Jin, W. Chen, and S. Zhang, "A Band-Divided Memory Polynomial for Wideband Digital Predistortion with Limited Bandwidth Feedback," IEEE Trans. Circuits Syst. II Express Briefs, vol. 62, no. 10, pp. 922-926, 2015.

[10] G. Ramsey, "Behavioral Modeling of Microwave Power Amplifiers Using a Look Up Table Method," Encycl. Inf. Sci. Technol. Third Ed., pp. 1242-1250, 2014.

[11] S. Yan, W. Shi, and J. Wen, "Review of neural network technique for modeling PA memory effect," 2016 IEEE MTT-S Int. Conf. Numer. Electromagn. Multiphysics Model. Optim. NEMO 2016, pp. 1-2, 2016.

[12] J. Staudinger, "DDR Volterra series behavioral model with fading memory and dynamics for high power infrastructure amplifiers," 2011 IEEE Radio Wirel. Week, RWW 2011 - 2011 IEEE Top. Conf. Power Amplifiers Wirel. Radio Appl. PAWR 2011, pp. 61-64, 2011.

[13] F. M. Ghannouchi, O. Hammi, and M. Helaoui, Behavioral Modeling and Predistortion of Wideband Wireless Transmitters. 2015 . 